\title{
BMJ Open Impact of bariatric surgery on neural food processing and cognition: an fMRI study
}

\author{
Marcel Schulze, ${ }^{1,2,3}$ Peter Sörös, ${ }^{1,4}$ Wolfgang Vogel, ${ }^{1}$ Thomas F Münte, ${ }^{5}$ \\ Helge H O Müller, ${ }^{3}$ Alexandra Philipsen ${ }^{1,3}$
}

To cite: Schulze M, Sörös $P$, Vogel W, et al. Impact of bariatric surgery on neural food processing and cognition: an fMRI study. BMJ Open 2018;8:e022375. doi:10.1136/ bmjopen-2018-022375

- Prepublication history for this paper is available online. To view these files, please visit the journal online (http://dx.doi org/10.1136/bmjopen-2018022375).

Received 14 February 2018 Revised 28 June 2018 Accepted 22 August 2018

Check for updates

(c) Author(s) (or their employer(s)) 2018. Re-use permitted under CC BY-NC. No commercial re-use. See rights and permissions. Published by BMJ.

${ }^{1}$ Medical Campus University of Oldenburg, School of Medicine and Health Sciences, Psychiatry and Psychotherapy, University Hospital, Oldenburg, Germany

${ }^{2}$ Section for Experimental Neuropsychiatry, Department of Psychiatry, Faculty of Medicine, University of Freiburg, Freiburg, Germany

${ }^{3}$ Department of Psychiatry and Psychotherapy, University of

Bonn, Bonn, Germany

${ }^{4}$ Research Center Neurosensory Science, University of

Oldenburg, Oldenburg, Germany

${ }^{5}$ Department of Neurology,

University of Luebeck, Luebeck, Germany

\section{Correspondence to}

Marcel Schulze;

marcel.schulze@uni-oldenburg. de

\section{ABSTRACT}

Introduction The Roux-en-Y gastric bypass (RYGB) is one of the most widely used techniques for bariatric surgery. After RYGB, weight loss up to $50 \%-70 \%$ of excess body weight, improvement of insulin-resistance, changes in food preferences and improvements in cognitive performance have been reported. This protocol describes a longitudinal study of the neural correlates associated with food-processing and cognitive performance in patients with morbid obesity before and after RYGB relative to lean controls.

Methods and analysis This study is a pre-post casecontrol experiment. Using functional MRI, the neural responses to food stimuli and a working memory task will be compared between 25 patients with obesity, pre and post RYGB, and a matched, lean control group. Resting state fMRI will be measured to investigate functional brain connectivity. Baseline measurements for both groups will take place 4 weeks prior to RYGB and 12 months after RYGB. The effects of RYGB on peptide tyrosine tyrosine and glucagon-like polypeptide-1 will also be determined. Ethics and dissemination The project has received ethical approval by the local medical ethics committee of the Carl-von-0ssietzky University of Oldenburg, Germany (registration: 2017-073). Results will be published in a peer-reviewed journal as original research and on international conferences.

Trial registration number DRKS00012495; Pre-results.

\section{BACKGROUND}

Obesity is defined as having a body mass index (BMI) of $\geq 30 \mathrm{~kg} / \mathrm{m}^{2}$. The prevalence of obesity has constantly been rising leading to significant health concerns since obesity is accompanied by health issues including diabetes mellitus, gallbladder disease, osteoarthritis, hypertension and cardiovascular diseases. $^{2} 3$ In 2016 worldwide, >1.9 billion adults were overweight of which $65 \%$ were obese. $^{4}$

The treatment of obesity can be roughly classified in three classes. Behavioural modification methods comprise revision of food consumption behaviour (eg, decreasing portion sizes and regular eating time), increasing the amount of physical activity,

\section{Strengths and limitations of this study}

- Employing functional MRI, the study will prospectively investigate neural plasticity of food processing and working memory following Roux-en-Y gastric bypass (RYGB).

- By including functional MRI, genetic analysis and behavioural measurements, RYGB-associated neural changes can be tracked longitudinally.

- Due to the longitudinal nature of the study, withdrawals of participants are expected, leading to participants with incomplete data.

analysing the lifestyle (and identification of trigger habits), as well as the identification of the emotional contents that might lead to imbalanced consumption behaviour. These measures can be supplemented by the use of medications such as lipase inhibitors, insulin sensitivity enhancer or modulators of hormone action (eg, glucagon-like polypeptide-1 (GLP-1) receptor agonist that stimulates insulin secretion). While deep brain stimulation of the nucleus accumbens has recently been described as being effective in morbid obesity in a case report, ${ }^{5}$ the only established invasive method to reduce weight is bariatric surgery. Surgical techniques aiming at weight loss comprise sleeve gastrectomy, gastric banding and gastric bypass surgery. ${ }^{6}$ Gastric bypass surgery is one of the most commonly employed weight loss techniques with average weight loss of around $50 \%-70 \%$ of excess body weight. ${ }^{78}$ The Roux-en-Y gastric bypass (RYGB) surgery is an irreversible procedure and is the most frequently applied bariatric technique. Here, the stomach is divided to create a small pouch which enables the food to bypass parts of the digestive tract. The pouch and the duodenum are reattached further down to the small intestine. ${ }^{9}$ After surgery, many patients show a marked decrease of weight, feel less hungry, eat less, show lower drive for food and are 
less preoccupied with food. ${ }^{10}$ Also, the food preferences change after surgery, which is reflected in lower ratings for high-fat and high-calorie food. ${ }^{11}$

One mechanism underlying the changes in food consumption behaviour after RYBG is altered expression of hormones, including the anorexigenic intestinal hormones peptide tyrosine tyrosine (PYY) and GLP-1. Both hormones show increased levels postprandially after surgery. ${ }^{1011}$ Therefore, the regulation of appetite is modulated through a delay in gastric emptying (through increased levels of PYY) and an increased glucose-dependent insulin release (through increased levels of GLP-1). ${ }^{12}$ In contrast, decreased levels of ghrelin, an orexigenic hormone showing stimulatory effects on food hedonics, were found after RYGB. ${ }^{11}{ }^{13}$ It has to be pointed out, however, that in about one-fifth of patients treated with bariatric surgery, the commonly accepted threshold of $50 \%$ excess weight loss (EWL) is not reached. ${ }^{14}$ This has particularly been attributed to intrapersonal factors such as external, reward-based eating behaviour and personality traits. ${ }^{1415}$ This calls for an investigation of the neural and behavioural changes of RYGB.

In functional MRI (fMRI) studies of the brain, changes in blood oxygen level-dependent (BOLD) signal for food stimuli have also been observed after RYGB. A postsurgical reduction in activation has been found within the mesolimbic pathway, that is, in areas which have been associated with reward behaviour. These include the ventral tegmental area, amygdala, hippocampus, anterior insula and ventral striatum. Interestingly, a selective reduction for high-energy versus low-energy food was observed, with greater reductions for high-energy food stimuli. ${ }^{10} 11$ Further, an increased sensitivity to salty taste and a decreased activation in the reward system for sweet taste has been reported. ${ }^{8}$ The mesolimbic pathway underlies the executive control of the prefrontal cortex, a structure responsible to initiate appropriate behavioural response to a given stimulus. ${ }^{16}$ Here, lower activations in the orbitofrontal cortex and medial prefrontal cortex have been reported after RYGB. ${ }^{1317}$ Of note, most previous studies showing changes in reward-related circuitry in patients undergoing RYBG have been performed within a relatively short interval between surgery and fMRI measurement of 4-12 weeks ${ }^{1618} 19$. As an exception, Wang et al studied the patients' neural responses to actual sweet and salty tastes 1 year after surgery. ${ }^{8}$ Despite well-documented behavioural effects of RYGB after 6years, a systematic long-term study investigating neural responses to food stimuli after 1 year of RYBG is lacking ${ }^{20}$ (see table 1 for an overview).

In addition to changes in hormone levels and brain circuitry underlying food intake control and processing, changes in cognitive functions after RYGB have also been reported. A key component contributing to cognitive performance are the executive functions $(\mathrm{EF})$, that is, metacognitive processes allowing regulation of behaviour towards a goal, self-regulation and decision-making. ${ }^{21} 22$ On a neural level in healthy participants, EF have been associated (among other areas) with activation in the dorsolateral prefrontal cortex (Brodmann Area (BA) 9, 46), anterior cingulate cortex (BA 32), superior and inferior parietal lobe (BA 7, 40), prefrontal cortex (BA 6, 10) and temporal cortex (BA 13). ${ }^{23}$ Obesity is associated with decreased EF performance, including attention and set shifting, inhibitory control, abstract reasoning, memory and visuospatial organisation. ${ }^{22} 2425$

Table 1 Overview of studies investigating the impact of RYGB on neuronal processing of food relevant items and cognition

\begin{tabular}{|c|c|c|c|c|}
\hline Author & $\begin{array}{l}\text { No. of patients- } \\
\text { controls } \\
\text { (M-F) }\end{array}$ & $\begin{array}{l}\text { Age (SD) } \\
\text { (patients-controls) }\end{array}$ & fMRI task & Pre/post interval \\
\hline $\begin{array}{l}\text { Frank et al } \\
(2016)^{7}\end{array}$ & $2(10)-6(6)$ & $\begin{array}{l}50 \pm 2.67 \\
50.7 \pm 3.29\end{array}$ & Food reward task & $\begin{array}{l}-/ \\
17.73 \pm 2.68 \text { months }\end{array}$ \\
\hline $\begin{array}{l}\text { Goldman et al } \\
(2013)^{54}\end{array}$ & $5(26)-0(0)$ & $45.87 \pm 11.08$ & Food craving/resisting task & $\begin{array}{l}-/ \\
3.07 \pm 2.0 \text { years }\end{array}$ \\
\hline $\begin{array}{l}\text { Ochner et al } \\
(2011)^{16}\end{array}$ & $0(10)-0(0)$ & $\begin{array}{l}35 \pm 9 \\
-\end{array}$ & Visual/auditory food stimulation & $\begin{array}{l}1 \text { month / } \\
1 \text { month }\end{array}$ \\
\hline $\begin{array}{l}\text { Van de Sande-Lee } \\
\text { et al }(2011)^{57}\end{array}$ & $2(11)-2(6)$ & $\begin{array}{l}34.0 \pm 10 \\
29.5 \pm 4\end{array}$ & D-glucose ingestion & $\begin{array}{l}0 / \\
\sim 8.3 \text { months }\end{array}$ \\
\hline $\begin{array}{l}\text { Wang et al } \\
(2016)^{8}\end{array}$ & $3(2)-5(2)$ & $\begin{array}{l}46.2 \pm 7.7 \\
51.7 \pm 7.8\end{array}$ & Taste testing (salt/sweet) & $\begin{array}{l}1 \text { month / } \\
12 \text { months }\end{array}$ \\
\hline
\end{tabular}


Additionally, cognitive processing speed is slowed, which has been observed in tasks such as the Stroop task, the controlled oral word association task and the digit symbol substitution task. ${ }^{22}$ In contrast, weight loss is associated with improvement in EF across different cognitive domains. ${ }^{26}$ One behavioural study reported improved working memory (WM) 12 weeks after bariatric surgery, which was reflected in an improved performance in the Digit Span test. ${ }^{24}$ Currently available studies showing the neural correlates of increased cognitive functioning after bariatric surgery are limited, though.

Changes in BOLD signal without the involvement of a task can be measured with resting state functional MRI (rs-fMRI). Correlated spontaneous fluctuations of the BOLD signal in different regions have been taken to indicate functional coupling of these regions to largescale networks and are generally denoted as 'functional connectivity'. ${ }^{27}$ Only one study reported changes in functional connectivity 1 year after RYGB surgery in patients with obesity. Here, a higher connectivity between regions involved in food-related saliency attribution and rewarddriven eating behaviour was found prior to surgery compared with lean controls. ${ }^{9}$ One year after bariatric surgery, changes in networks related to cognitive control over eating and bodily perception were reported. Despite a well-chosen experimental design this study suffered from a small sample size and did not provide a psychological profile of the participants. To further elucidate the change in functional connectivity associated with RYGB, the current study will include rs-fMRI.

\section{STUDY AIMS}

The planned study will examine neural changes associated with RYGB. We hypothesise that, prior to RYGB, obese individuals show increased neuronal activity to food cues in reward-related brain areas compared with a healthy, lean control group. The enhanced food processing is expected to be reduced 12 months postsurgery. Hormonal status will also be affected by RYGB; we expect lower PYY and GLP-1 levels, compared with healthy individuals, prior to surgery with a respective change after 12 months. The hormonal status of PYY and GLP-1 are expected to be negatively correlated with the neuronal response in reward-related brain areas to food images. We hypothesise that lower hormonal levels are negatively correlated to a heightened brain response in reward-related areas to food stimuli prior to RYGB. Respectively, we expect higher hormonal levels are negatively correlated with a lowered brain response in reward areas to food stimuli 12 month after RYGB. We expect a lower performance in WM in obese subjects compared with healthy controls. After RYGB, we assume a significant increase in WM performance in the bariatric group, which is also reflected on the neuronal level by enhanced recruitment of brain areas associated with executive functioning. Compared with controls, functional connectivity is expected to differ in participants with obesity
Box 1 Overview of the hypotheses and main outcome variables

- H1: patients with obesity show enhanced activity in reward-related brain areas to food cues prior to RYGB compared with healthy controls.

- H2: 12 months after RYGB, patients show a decrease in neural responses to food cues compared with pre-surgery.

- H3: working memory performance will be lower in patients with obesity prior to RYGB compared with healthy controls, which is reflected in lower prefrontal activation.

- H4: working memory performance will be improved 12 months after RYGB in patients with obesity, which is reflected in increased prefrontal activation.

- H5: RYGB leads to a decrease in functional connectivity, in particular, in the salience network.

- H6: hormonal status is negatively correlated with the neural response in reward-related brain areas to food images.

- Primary outcome variables: neural processing of food stimuli, cognitive performance during the 2-back task and functional connectivity.

- Secondary outcome variables: hormonal status and behavioural data (derived from questionnaires).

RYGB, Roux-en-Y gastric bypass

prior to RYGB in terms of a stronger connectivity in the salience network with diminishing effects at the 12-month follow-up measurement (see box 1).

\section{METHODS}

\section{Participants}

In total, 50 female participants (25 patients with obesity and 25 lean control subjects) aged from 18 to 60 years will be recruited at the Centre for Obesity in Friesoythe, Germany and at the Carl-von-Ossietzky University Oldenburg, Germany. The patients with obesity will be screened for suitability for bariatric surgery according to the German guideline for prevention and therapy of obesity ${ }^{28}$ by an experienced surgeon (WV). Decision for treatment will be made independent of the current study. Anxiety will be no exclusion criterion since there is a high prevalence in people with obesity. ${ }^{29}$ Participants with obesity need to have a BMI $>35 \mathrm{~kg} / \mathrm{m}^{2}$ to get enrolled in the study. Obesity-related comorbidities such as diabetes mellitus, hypertension, sleep apnoea and dyslipidaemia will be no exclusion criteria. Inclusion criteria for the lean, healthy control group are a BMI $<25 \mathrm{~kg} / \mathrm{m}^{2}$, no history of any psychiatric disorder and no history of eating disorder. Exclusion criteria are substance abuse including nicotine, current major depression, psychopharmalogical treatment, neurological disorders, pregnancy, claustrophobia and MRI contraindications (eg, metallic implants and cardiac pacemaker). The control group will be matched to the obese group for age, sex and education. Since the study will only enrol female participants, the menstrual cycle will be controlled for by assessing only in the midfollicular phase (days 4-8 after onset of menses) due to 
differences in brain responses to reward between the follicular and the luteal phase. ${ }^{30}$

\section{Patient and public involvement}

Patients were not involved in the development of the research question, outcome measures or study design.

\section{Behavioural assessment}

At the baseline measurement (up to 4 weeks prior to RYGB) and at the follow-up measurement (between 12 and 13 months after RYGB), the participants will be asked to fill out a demographic questionnaire and the Freiburger Persönlichkeitsinventar (Freiburg Personality Inventory, FPI-R) ${ }^{31}$ The FPI-R is a personality test, which assesses personality traits on 12 scales. To assess psychiatric comorbidity, the structured clinical interview for DSM-V will be administered (SKID-I/II, German version). ${ }^{32}$

The participants will be in a fasting period for 4 hours before the measurement. All the measurements will take place at around the same time of the day, which will be between $3 \mathrm{pm}$ and $5 \mathrm{pm}$. The current state of hunger at time of the fMRI will be assessed on a 10-point Likert scale. Additionally, the time of the previous meal and the portion size of the previous meal will be recorded. To have a measurement on food consumption before the fMRI recording, the participants will be asked to complete a food diary 1 week prior to the pre/post recording in order to document what has been eaten, at which time of the day and also how much was eaten. The Fragebogen zum Essverhalten (Questionnaire of Eating Behaviour, FEV) will be administered. ${ }^{33}$ The FEV measures the cognitive control of the eating behaviour, how impulsive the eating behaviour is and how the feeling of hunger is perceived. For assessing depressive symptoms, the German version of the Beck Depression Inventory (BDI) will be administered. ${ }^{34}$ Abnormal eating behaviour and the inability to control the overconsumption of food has been linked to impulsivity. Moreover, it was found that impulsivity measures can predict weight reduction in patients undergoing bariatric surgery. ${ }^{35}$ Therefore, all participants will be asked to fill out the German version of the Behavioural Inhibition System (BIS), a questionnaire examining impulsivity. ${ }^{36}$ Moreover, there is meta-analytic evidence for a significant association between obesity and attention-deficit/hyperactivity disorder (ADHD). ${ }^{37}$ To assess ADHD symptoms, the German versions of the Wender-Utah Rating scale and the Conners' adult ADHD self-rating scales will be applied (CAARS) ${ }^{38}{ }^{39}$ To control for a possible change in food preferences due to RYGB, the participants will rate the liking and wanting of each food image after the fMRI task on a 5-point Likert scale. Also, to have a measurement on weight after RYGB, the participants need to record the body weight on a weekly basis during the pre-post interval.

\section{Medical assessment}

Obesity-related comorbidities will be recorded. These include diabetes mellitus, hypertension, sleep apnoea and dyslipidaemia. Besides the BMI, the waist-to-hip-ratio will be measured and a body composition analysis will be done. These procedures together address fat distribution which is not provided by the BMI alone. ${ }^{4041}$

\section{Blood sample}

A venous blood sample will be collected. The sample will then be centrifuged and the plasma will be aliquoted and stored at $-80^{\circ} \mathrm{C}$. The plasma concentrations of PYY and GLP-1 will be determined using standardised methods. Important, the blood sample will be collect at the same time point to prevent circadian influences. The participants will be asked not to eat 4 hours before the blood sample collection.

\section{fMRI task}

Each participant undergoes a $\sim 1$ hour MRI session at baseline (up to 4 weeks prior to RYGB) and at the follow-up time point (between 12 and 13 months after RYGB).

\section{Food stimuli processing}

During fMRI, participants will be required to watch images that show food or non-edible objects (eg, tools). Stimuli were taken from an existing and validated image database for experimental research on eating and appetite. ${ }^{42}$ To account for possible differences on arousal and valence between pictures, we did an in-house rating: each picture was rated by 20 women with the self-assessment manikin on the scales arousal and valence. ${ }^{43}$ Further, the food pictures were rated either as high- or low-caloric. These images have been used in previous neuroimaging studies (eg, Blechert et al). ${ }^{44}$ In total, 190 food images and 190 neutral images will be shown, split in two runs containing 95 food and 95 object images, each. These images will be shown in a pseudorandomised order of 13 blocks containing 15 images of food items and in 13 blocks of 15 images of non-edible objects. Within each block, each image will be presented for $1 \mathrm{~s}$ with an interstimulus interval (ISI) of $200 \mathrm{~ms}$. We decided to use a block design fMRI experiment with $1 \mathrm{~s}$ of image presentation and a brief ISI to maximise the neural responses to the visual stimuli. Our stimulus duration of $1 \mathrm{~s}$ is shorter than the stimulus duration used for example, by Blechert et $a t^{44}$ who presented their images for $2.5 \mathrm{~s}$ in an event-related paradigm. The shorter stimulus duration of $1 \mathrm{~s}$ helps to avoid excessive eye movement. To ensure attention, participants will be asked whether a certain item was present after 15 images, followed by $10 \mathrm{~s}$ of baseline (black screen with fixation cross, see figure 1).

\section{Desire for food}

To assess food preferences at an explicit level, participants will perform a modified version of the approach-avoidance task. ${ }^{45} 46$ Here, high-caloric and low-caloric food images will be presented. A sense of approaching or avoiding will be created by the participants' response to an image via a button press. By wanting a specific food item, the image will grow bigger and get smaller when the item is unwanted by the participant. First, 10 practice 
A)

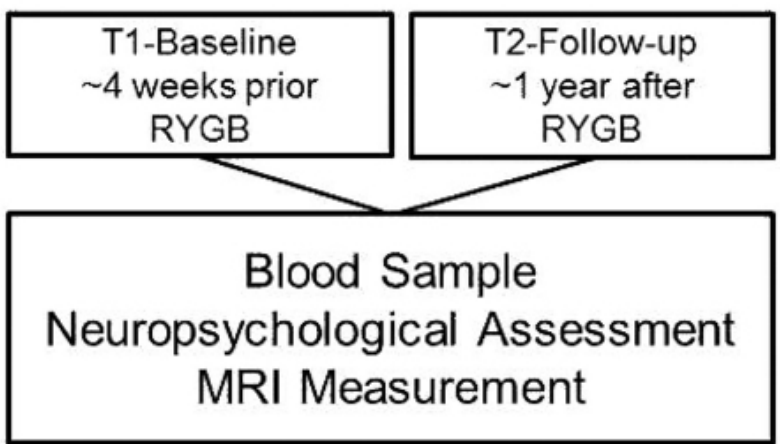

C)

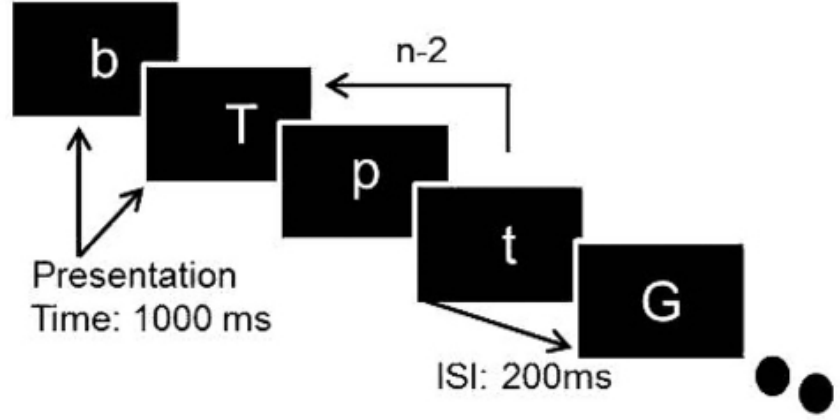

B)

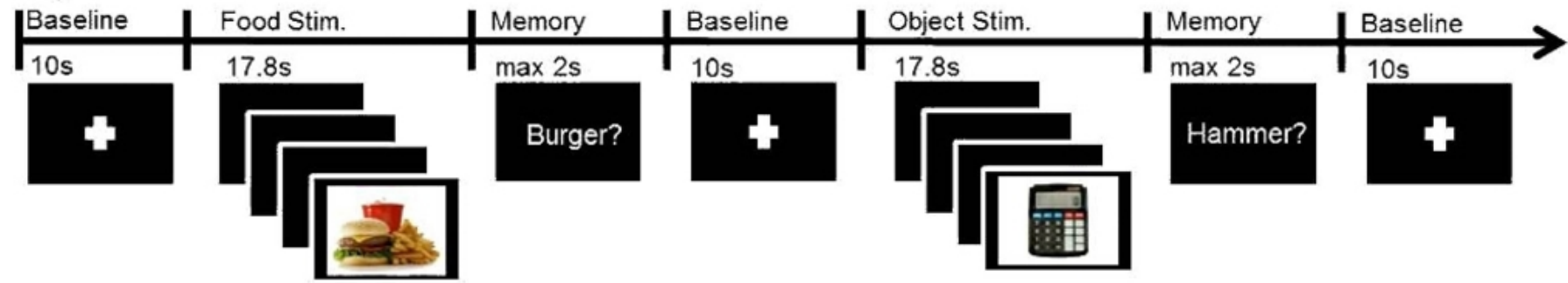

Figure 1 Schematic overview of (A) study timeline; (B) food stimulation paradigm; (C) 2-back task. ISI, interstimulus interval.

trials will be performed with neutral objects, followed by the presentation of 30 high-caloric and 30 low-caloric food images in a pseudorandomised order. Participants will be asked to respond as quickly and accurately as possible. The image will disappear once the item will be at largest or smallest size determined by the participants' response.

\section{Working memory}

Participants will be performing a letter version of the n-back task. The task demands the maintenance and permanent updating of new information in WM. It is required to observe a series of stimuli and to respond as soon as possible whenever a stimulus is presented that is the same as the one presented two trials before (2-back). ${ }^{47}$ To reduce phonological and visual strategies, phonologically similar letters will be presented in lower and upper case: b, B, d, D, g, G, p, P, t, T, v, V. The case of the letters has to be ignored. The participants have to respond with a button press for a hit. Two blocks with a total duration of $14 \mathrm{~min}$ will be pseudorandomised with a white letter presentation on a black background. Each letter will be presented for $1 \mathrm{~s}$ and an ISI of $500 \mathrm{~ms}$. In order to ensure a proper understanding of the task, participants will practice the 2-back task outside the scanner (figure 1).

\section{Resting state}

Resting state fMRI will be measured for $9 \mathrm{~min}$. The participants will be instructed to not focus on specific thoughts and remain still with eyes open. The participants will be instructed to fixate a cross during the recording.

\section{MRI data acquisition}

Whole-brain fMRI blood oxygen-level dependent data will be acquired on a $3 \mathrm{~T}$ Siemens Magnetom Prisma with a 64-channel head coil at the Neuroimaging Unit, University of Oldenburg (https://www.uni-oldenburg. de/neuroimaging/) using an echo-planar T2*-weighted imaging sequence. A high-resolution T1-weighted anatomical image will be also be obtained. To ensure the absence of structural abnormalities in the brain, an experienced MRI technologist will examine all structural images. In case of an abnormality, we will contact a radiologist for further diagnostic scanning.

\section{MRI data analysis}

Analysis of the data will be carried out using fMRI Expert Analysis Tool), part of FSL (FMRIB's software Library, www.fmrib.ox.ac.uk/fsl). ${ }^{48}$ For preprocessing, functional data will be motion corrected, temporally filtered with a high-pass filter and spatially smoothed using a Gaussian kernel. For each condition (visual stimulation: food images; object images; n-back task: hits, misses and false alarms), the BOLD response will be modelled using separate explanatory variables $(\mathrm{EV})$. Each $\mathrm{EV}$ will be convolved with a canonical haemodynamic response function to model the haemodynamic response. Some nuisance covariates such as head motion parameters, global mean signal, white matte signal and cerebrospinal fluid signal will be regressed out to reduce the signal of no interest. Finally, individual data will be fitted to a general linear model as implemented in FSL. Non-linear registration to the MNI152 standard space template will be realised using FMRIB's Linear Image Registration Tool 
and Non-linear Image Registration Tool. Group statistics will be carried out using FMRIB's Local Analysis of Mixed Effects stage 1 and stage 2 with automatic outlier de-weighting. For correction of multiple comparisons, clusters will be thresholded at $\mathrm{Z}=3.1 \quad(\mathrm{p}<0.05)$. Repeated measured analysis of variance (ANOVA) with the factors group, time and condition will be used to determine group differences in food stimuli processing, approach avoidance task and 2-back task performance.

Resting state fMRI will be analysed with the seed-tovoxel method using the toolbox CONN (http://www. conn-toolbox.org) and with independent component analysis using dual regression as implemented in FSL. ${ }^{49}$ For the seed-to-voxel analysis, regions of interest will be chosen a priori based on the findings of relevant earlier studies (eg, salience network). Associations between the time courses of the fMRI signal in these seed regions and in all other voxels of the brain will be computed. For dual regression, a model-free approach based on FSL's MELODIC will be used. ${ }^{50}$

All resting state datasets will be decomposed into sets of time courses and associated spatial maps which describe the temporal and spatial characteristics of underlying hidden signals. ${ }^{51}$ This procedure does not only enable removal of artefacts (eg, scanner drift) but also the isolation of neural networks, which results in template maps. Once the group ICA templates are created, they are regressed against the individual spatial maps (spatial regression) in order to identify the subject-specific time courses. To identify the subject-specific spatial maps, the network-specific time courses from the first regression step are used as predictors in a second regression stage. ${ }^{49}$ The subject-specific time courses and spatial maps will be compared between the RYGB group and the control group, as well as between the pre and post intervention measurements.

\section{Behavioural data analysis}

Statistical analysis will be performed using SPSS (V.24, 2013). If the data are normally distributed, we plan to perform repeated measures ANOVA with the factors group $\mathrm{X}$ time which will be used to determine group and time differences for the demographic, psychometric and neuropsychological data. A linear regression will be performed with the test scores to clarify whether there has been an influence of the finding in the behavioural assessment on performance during food processing, approach avoidance task, and 2-back task. The test scores will be normalised to z-scores for statistical analysis with imaging data. Differences in hormonal status will be presumably evaluated with Student's t-tests, given that the data are normal distributed.

\section{Sample size justification}

The planned study will compare the neuronal response of participants with obesity with a healthy control group. Besides the group comparison, within group comparison will also be obtained. A study sample of 25 in an
fMRI measurement leads to sufficient power as it is shown in Thirion et al. ${ }^{52}$ Using G*Power (http://www. gpower.hhu.de/) to estimate the effect size, based on a repeated measures ANOVA with within-between interaction $(\alpha=0.05)$ yield in a medium effect size $(0.36)$ with a power of $0.95 .^{53}$

\section{Value of the study}

The current study, with its longitudinal design, will add information about the neuronal correlates of food processing before and after a RYGB to the existing literature. In particular, it is unknown so far whether RYGB leads to long-term adjustments in the neural responses to food and to lasting improvements of cognitive functions. This gap will be closed by food processing and WM tasks of the current study.

\section{ETHICS AND DISSEMINATION}

The study will be conducted in accordance with the Declaration of Helsinki in order to ensure the well-being and rights of the participants. The project has received ethical approval by the local medical ethics committee of the Carl-von-Ossietzky University of Oldenburg (registration: 2017-073). Written informed consent will be obtained from all participants. Participants will be able to withdraw from the study at any time without giving any reasons. During all measurement, medical professionals will be present. The study is registered at the German Clinical Trial Register with the trial registration number: DRKS00012495. Presumably, the recruitment of the participants will be finished by July 2019 .

Contributors MS, WV, PS, TM and AP: were involved in the design of the protocol for the study. MS: wrote the first draft of this manuscript. WV, PS, TM, HM and AP: critically contributed to and redefined this manuscript. All authors approved the final manuscript.

Funding The authors have not declared a specific grant for this research from any funding agency in the public, commercial or not-for-profit sectors.

Competing interests None declared.

Patient consent Not required.

Ethics approval Medical Ethics Committee University of Oldenburg.

Provenance and peer review Not commissioned; externally peer reviewed.

Data sharing statement The authors presumably agree on data sharing in the result article.

Open access This is an open access article distributed in accordance with the Creative Commons Attribution Non Commercial (CC BY-NC 4.0) license, which permits others to distribute, remix, adapt, build upon this work non-commercially, and license their derivative works on different terms, provided the original work is properly cited, appropriate credit is given, any changes made indicated, and the use is non-commercial. See: http://creativecommons.org/licenses/by-nc/4.0/.

\section{REFERENCES}

1. $\mathrm{Ng} \mathrm{M}$, Fleming $\mathrm{T}$, Robinson $\mathrm{M}$, et al. Global, regional, and national prevalence of overweight and obesity in children and adults during 1980-2013: a systematic analysis for the Global Burden of Disease Study 2013. Lancet 2014;384:766-81.

2. Bray GA. Medical consequences of obesity. J Clin Endocrinol Metab 2004;89:2583-9. 
3. Heymsfield SB, Wadden TA. Mechanisms, pathophysiology, and management of obesity. N Engl J Med 2017;376:254-66.

4. Organization WH. Obesity and Overweight factsheet from the WHO: Organization WH, 2017.

5. Tronnier VM, Rasche D, Thorns V, et al. Massive weight loss following deep brain stimulation of the nucleus accumbens in a depressed woman. Neurocase 2018;24:49-53.

6. Rosenblum J, Venkatesh RD. The Massgeneral hospital for children adolescent medicine handbook, 2017:67-75.

7. Frank S, Heinze JM, Fritsche A, et al. Neuronal food reward activity in patients with type 2 diabetes with improved glycemic control after bariatric surgery. Diabetes Care 2016;39:1311-7.

8. Wang JL, Yang Q, Hajnal A, et al. A pilot functional MRI study in Roux-en-Y gastric bypass patients to study alteration in taste functions after surgery. Surg Endosc 2016;30:892-8.

9. Olivo G, Zhou W, Sundbom M, et al. Resting-state brain connectivity changes in obese women after Roux-en-Y gastric bypass surgery: a longitudinal study. Sci Rep 2017;7:6616.

10. Behary P, Miras AD. Food preferences and underlying mechanisms after bariatric surgery. Proc Nutr Soc 2015;74:419-25.

11. Scholtz S, Miras AD, Chhina N, et al. Obese patients after gastric bypass surgery have lower brain-hedonic responses to food than after gastric banding. Gut 2014;63:891-902.

12. De Silva A, Bloom SR. Gut Hormones and Appetite Control: a focus on PYY and GLP-1 as therapeutic targets in obesity. Gut Liver 2012;6:10-20.

13. Faulconbridge LF, Ruparel K, Loughead J, et al. Changes in neural responsivity to highly palatable foods following roux-en-Y gastric bypass, sleeve gastrectomy, or weight stability: An fMRI study. Obesity 2016;24:1054-60.

14. Sarwer DB, Wadden TA, Fabricatore AN. Psychosocial and behavioral aspects of bariatric surgery. Obes Res 2005;13:639-48.

15. Agüera Z, García-Ruiz-de-Gordejuela A, Vilarrasa N, et al. Psychological and personality predictors of weight loss and comorbid metabolic changes after bariatric surgery. Eur Eat Disord Rev 2015;23:509-16.

16. Ochner CN, Kwok Y, Conceição E, et al. Selective reduction in neural responses to high calorie foods following gastric bypass surgery. Ann Surg 2011;253:502-7

17. Goldstone AP, Miras AD, Scholtz S, et al. Link between increased satiety gut hormones and reduced food reward after gastric bypass surgery for obesity. J Clin Endocrinol Metab 2016;101:599-609.

18. Bruce AS, Bruce JM, Ness AR, et al. A comparison of functional brain changes associated with surgical versus behavioral weight loss. Obesity 2014;22:337-43.

19. Frank $S$, Wilms $B$, Veit $R$, et al. Altered brain activity in severely obese women may recover after Roux-en $\mathrm{Y}$ gastric bypass surgery. Int $\mathrm{J}$ Obes 2014;38:341-8.

20. Adams TD, Davidson LE, Litwin SE, et al. Health benefits of gastric bypass surgery after 6 years. JAMA 2012;308:1122-31.

21. Stuss DT. Functions of the frontal lobes: relation to executive functions. J Int Neuropsychol Soc 2011;17:759-65.

22. Sweat V, Yates KF, Migliaccio R, et al. Obese adolescents show reduced cognitive processing speed compared with healthy weight peers. Child Obes 2017;13:190-6.

23. Kim NY, Wittenberg E, Nam CS. Behavioral and neural correlates of executive function: interplay between inhibition and updating processes. Front Neurosci 2017;11:378.

24. Alosco ML, Spitznagel MB, Strain G, et al. Improved memory function two years after bariatric surgery. Obesity 2014;22:32-8.

25. Prickett C, Brennan L, Stolwyk R. Examining the relationship between obesity and cognitive function: a systematic literature review. Obes Res Clin Pract 2015;9:93-113.

26. Veronese N, Facchini S, Stubbs B, et al. Weight loss is associated with improvements in cognitive function among overweight and obese people: a systematic review and meta-analysis. Neurosci Biobehav Rev 2017;72:87-94.

27. Birn RM, Molloy EK, Patriat R, et al. The effect of scan length on the reliability of resting-state fMRI connectivity estimates. Neuroimage 2013;83:550-8

28. Hauner H, Moss A, Berg A, et al. Interdisziplinäre leitlinie der qualität s3 zur „prävention und therapie der adipositas “. Adipositas 2014:8:179-221.

29. Evers SS, Sandoval DA, Seeley RJ. The physiology and molecular underpinnings of the effects of bariatric surgery on obesity and diabetes. Annu Rev Physiol 2017;79:313-34.
30. Dreher JC, Schmidt PJ, Kohn P, et al. Menstrual cycle phase modulates reward-related neural function in women. Proc Natl Acad Sci U S A 2007;104:2465-70.

31. Fahrenberg J, Hampel R, Fpi-r SH. Das Freiburger Persönlichkeitsinventar Manual, 2001.

32. First M, Williams J, Karg R, et al. Structured clinical interview for DSM-5 disorders, clinician version (SCID-5-CV. Arlington, VA: American Psychiatric Association, 2015.

33. Pudel V, Westenhöfer J. Fragebogen zum essverhalten (FEV). handanweisung: Verlag für Psychologie: Hogrefe, 1989.

34. Beck AT, Steer RA, Brown GK. Beck depression inventory-II. San Antonio 1996;78:490-8.

35. Kulendran M, Borovoi L, Purkayastha S, et al. Impulsivity predicts weight loss after obesity surgery. Surg Obes Relat Dis 2017;13:1033-40.

36. Jorm AF, Christensen H, Henderson AS, et al. Using the BIS/BAS scales to measure behavioural inhibition and behavioural activation: Factor structure, validity and norms in a large community sample. Pers Individ Dif 1998;26:49-58.

37. Cortese S, Moreira-Maia CR, St Fleur D, et al. Association between ADHD and obesity: a systematic review and meta-analysis. Am $J$ Psychiatry 2016;173:34-43.

38. Conners' adult ADHD rating scales (CAARS). Technical manual. MHS North Tonawanda, 1999.

39. Retz-Junginger P, Retz W, Blocher D, et al. Reliabilitt und Validitt der Wender-Utah-Rating-Scale-Kurzform. Nervenarzt 2003;74:987-93.

40. Müller MJ, Geisler C. Defining obesity as a disease. Eur J Clin Nutr 2017;71:1256-8.

41. Müller MJ, Braun W, Enderle J, et al. Beyond BMl: conceptual issues related to overweight and obese patients. Obes Facts 2016:9:193-205.

42. Blechert J, Meule A, Busch NA, et al. Food-pics: an image database for experimental research on eating and appetite. Front Psychol 2014;5:617.

43. Bradley MM, Lang PJ. Measuring emotion: the self-assessment manikin and the semantic Differential. J Behav Ther Exp Psychiatry 1994;25:49-59.

44. Blechert J, Klackl J, Miedl SF, et al. To eat or not to eat: effects of food availability on reward system activity during food picture viewing. Appetite 2016;99:254-61.

45. Wiers R, Rinck M, Dictus M, et al. Relatively strong automatic appetitive action-tendencies in male carriers of the OPRM1 G-allele. Genes. Brain and Behavior 2009;8:101-6.

46. Wiers RW, Eberl C, Rinck M, et al. Retraining automatic action tendencies changes alcoholic patients' approach bias for alcohol and improves treatment outcome. Psychol Sci 2011;22:490-7.

47. Owen AM, McMillan KM, Laird AR, et al. N-back working memory paradigm: a meta-analysis of normative functional neuroimaging studies. Hum Brain Mapp 2005;25:46-59.

48. Smith SM, Jenkinson M, Woolrich MW, et al. Advances in functional and structural MR image analysis and implementation as FSL. Neuroimage 2004;23 Suppl 1:S208-S219.

49. Nickerson LD, Smith SM, Öngür D, et al. Using dual regression to investigate network shape and amplitude in functional connectivity analyses. Front Neurosci 2017;11:11.

50. Beckmann CF. Modelling with independent components. Neuroimage 2012;62:891-901.

51. Beckmann CF, DeLuca M, Devlin JT, et al. Investigations into restingstate connectivity using independent component analysis. Philos Trans R Soc Lond B Biol Sci 2005;360:1001-13.

52. Thirion $B$, Pinel $P$, Mériaux $S$, et al. Analysis of a large $f M R I$ cohort: statistical and methodological issues for group analyses. Neuroimage 2007;35:105-20.

53. Faul F, Erdfelder E, Lang AG, et al. G*Power 3: a flexible statistical power analysis program for the social, behavioral, and biomedical sciences. Behav Res Methods 2007;39:175-91.

54. Goldman RL, Canterberry M, Borckardt JJ, et al. Executive control circuitry differentiates degree of success in weight loss following gastric-bypass surgery. Obesity 2013;21:2189-96.

55. Ochner CN, Laferrère B, Afifi L, et al. Neural responsivity to food cues in fasted and fed states pre and post gastric bypass surgery. Neurosci Res 2012;74:138-43.

56. Scholtz S, Miras AD, Chhina N, et al. Obese patients after gastric bypass surgery have lower brain-hedonic responses to food than after gastric banding. Gut 2014;63.

57. van de Sande-Lee S, Pereira FR, Cintra DE, et al. Partial reversibility of hypothalamic dysfunction and changes in brain activity after body mass reduction in obese subjects. Diabetes 2011;60:1699-704. 